\title{
Long-Term Outcomes after Early Neonatal Hyperglycemia in VLBW Infants: A Systematic Review
}

\author{
Megan E. Paulsen ${ }^{a}$ Sarah Jane Brown ${ }^{b}$ Katherine M. Satrom ${ }^{a}$ \\ Johannah M. Scheurer ${ }^{\text {a }}$ Sara E. Ramel ${ }^{\text {a }}$ Raghavendra B. Rao ${ }^{\text {a }}$

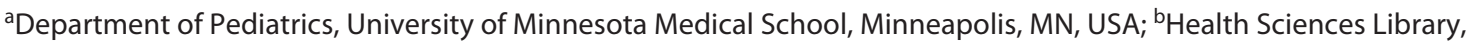 \\ University of Minnesota, Minneapolis, MN, USA
}

\section{Keywords}

Glucose $\cdot$ Insulin · Preterm infant · Prognosis .

Neurodevelopment · Metabolic · Neonatal intensive care unit

\begin{abstract}
Introduction: Long-term effects of early hyperglycemia in VLBW infants are poorly characterized. The objective of this study was to systematically review the effect of early hyperglycemia on growth, metabolic health, and neurodevelopment after neonatal intensive care unit discharge in VLBW infants. Methods: The systematic review was conducted in accordance with the PRISMA guidelines. A study protocol was registered in PROSPERO (CRD42019123335). Data sources included Ovid MEDLINE, Embase, Cochrane Library, Cl$\mathrm{NAHL}$, and Scopus. Selected studies included infants with a blood glucose concentration $>150 \mathrm{mg} / \mathrm{dL}$ (8.3 mmol/L) during the first 28 days of life, a gestational age (GA) $<32$ weeks, and/or a birth weight $<1,500 \mathrm{~g}$ and longitudinal data on growth, metabolic health, or neurodevelopment outcomes. The GRADE system was used to assess quality of evidence. Results: Eight studies ( $n=987$ infants) reported long-term outcomes from 4-month corrected GA to 7 years old. Most
\end{abstract} \\ Karger'}

studies compared long-term outcomes of preterm infants with and without hyperglycemia. Two studies addressed outcomes related to interventions following early hyperglycemia. Some studies found differences in growth, metabolic health, and neurodevelopment outcomes between VLBW preterm infants with hyperglycemia and without hyperglycemia, while other studies found no differences between groups. The overall graded quality of evidence was low. Conclusions: Well-designed randomized controlled and prospective studies are necessary to determine the effect of early hyperglycemia and its treatment on later metabolic and neurodevelopmental outcomes in VLBW infants. Authors propose a potential study design for standardizing the assessment of long-term metabolic and neurodevelopmental outcomes following early hyperglycemia in preterm infants.

(c) 2021 S. Karger AG, Basel

\section{Introduction}

Very low birth weight (VLBW) infants defined as infants born with a birth weight $<1,500 \mathrm{~g}$, frequently develop hyperglycemia soon after birth [1-7]. Studies using continuous glucose monitoring report the incidence of
Correspondence to:

Megan E Paulsen, mgosslin@umn.edu 
Table 1. Population-exposure-comparison-outcome-time summary statement

\begin{tabular}{ll}
\hline Study population & VLBW infants $(<32$ weeks or $<1,500 \mathrm{~g})$ \\
\hline Exposure & Hyperglycemia \pm insulin treatment \\
Comparison & Normoglycemic/standard glycemic control/noninsulin treatment \\
Outcomes & Growth, metabolic, and neurodevelopmental outcomes \\
Time & Studies published through August 30, 2020 \\
Setting & Worldwide \\
Study design & Any study design with longitudinal data and at least 1 post-NICU follow-up \\
\hline
\end{tabular}

VLBW, very low birth weight.

early hyperglycemia between 43 and $80 \%$ in VLBW infants $[2,8]$. The prevalence of hyperglycemia is inversely correlated with birth weight and gestational age (GA) [2]. The short-term effects of hyperglycemia in VLBW infants are more frequently reported in the literature than the long-term effects $[4,7,9-11]$. Long-term risks associated with hyperglycemia in VLBW infants are studied less and therefore poorly characterized. Outcomes extrapolated from infants of diabetic mothers, a population exposed to hyperglycemia during a similar period of development, suggest that hyperglycemia may affect long-term metabolic and neurodevelopmental outcomes [12-15].

Hyperglycemia in the very preterm population is a biochemical disorder representing an imbalance between excess available glucose and decreased glucose utilization [16]. Dextrose infusion for enhanced nutrition, relative insulin resistance, defective islet $\beta$-cell function, and aberrant counter-regulatory hormones are the most common etiologies of hyperglycemia in preterm infants [16, 17]. Hyperglycemia can be further induced or exacerbated in VLBW infants by common neonatal intensive care unit (NICU) morbidities such as sepsis [18]. Hyperglycemia causes increased fat deposition, reduced lean mass accretion, pancreatic $\beta$-cell toxicity, fatty infiltration of the heart and liver, and cardiac septal hypertrophy and RV dysfunction [16]. Hyperglycemia also impairs neuronal development with fewer dendrites and reduced synapse formation in animal models [16]. These physiologic complications of hyperglycemia provide biologic plausibility for the development of metabolic and neurodevelopmental complications following early hyperglycemia in the VLBW population.

The objective of this systematic analysis was to answer the following question: is there enough evidence on longterm outcomes to guide clinical management of hyperglycemia in VLBW infants? We reviewed all relevant published studies that evaluated long-term growth, metabol- ic, and neurodevelopmental outcomes after early hyperglycemia in VLBW infants. Following a review of the relevant articles, we assessed the quality of evidence to determine if there were associations between (1) hyperglycemia and long-term growth, metabolic, and neurodevelopment outcomes; (2) severity and duration of hyperglycemia and long-term outcomes; and (3) treatment and long-term outcomes.

\section{Methods}

The systematic review was conducted in accordance with the PRISMA guidelines (see online suppl. 1; for all online suppl. material, see www.karger.com/doi/10.1159/000517951) [19]. A study protocol was registered in PROSPERO (CRD42019123335) on https://www.crd.york.ac.uk/prospero. The review questions were formatted in the PICOTT format (Table 1) [20].

\section{Search Strategy}

The search strategy was built and tested using Ovid MEDLINE, included both MeSH terms and keywords (online suppl. 2), and translated to 4 other databases as follows: Embase, Cochrane Library, CINAHL, and Scopus. No search limits were applied with respect to publication date or language. Search results were deduplicated using EndNote. Hand searching methods, including searching reference lists of articles selected for inclusion, were also used to identify relevant materials that may have been missed by the database search. Only full-text publications were included in the analysis. Authors of abstracts were contacted to determine if full-text publications existed prior to manuscript submission.

\section{Inclusion Criteria}

Any study design was included. The population of interest was infants admitted to the NICU worldwide who were born at $<32$ weeks GA and/or were VLBW. Criteria for study inclusion were as follows: (1) blood glucose (BG) concentrations of $>150 \mathrm{mg} / \mathrm{dL}(8.3$ $\mathrm{mmol} / \mathrm{L}$ ) in the first 28 days after birth and (2) a GA $<32$ weeks or a birth weight $<1,500 \mathrm{~g}$. Titles and abstracts of reports from the above search strategy were assessed independently by 2 review authors using Rayyan to determine their eligibility for inclusion [21]. Disagreements were resolved by consensus. If consensus could not 
Fig. 1. PRISMA systematic review flow dia-

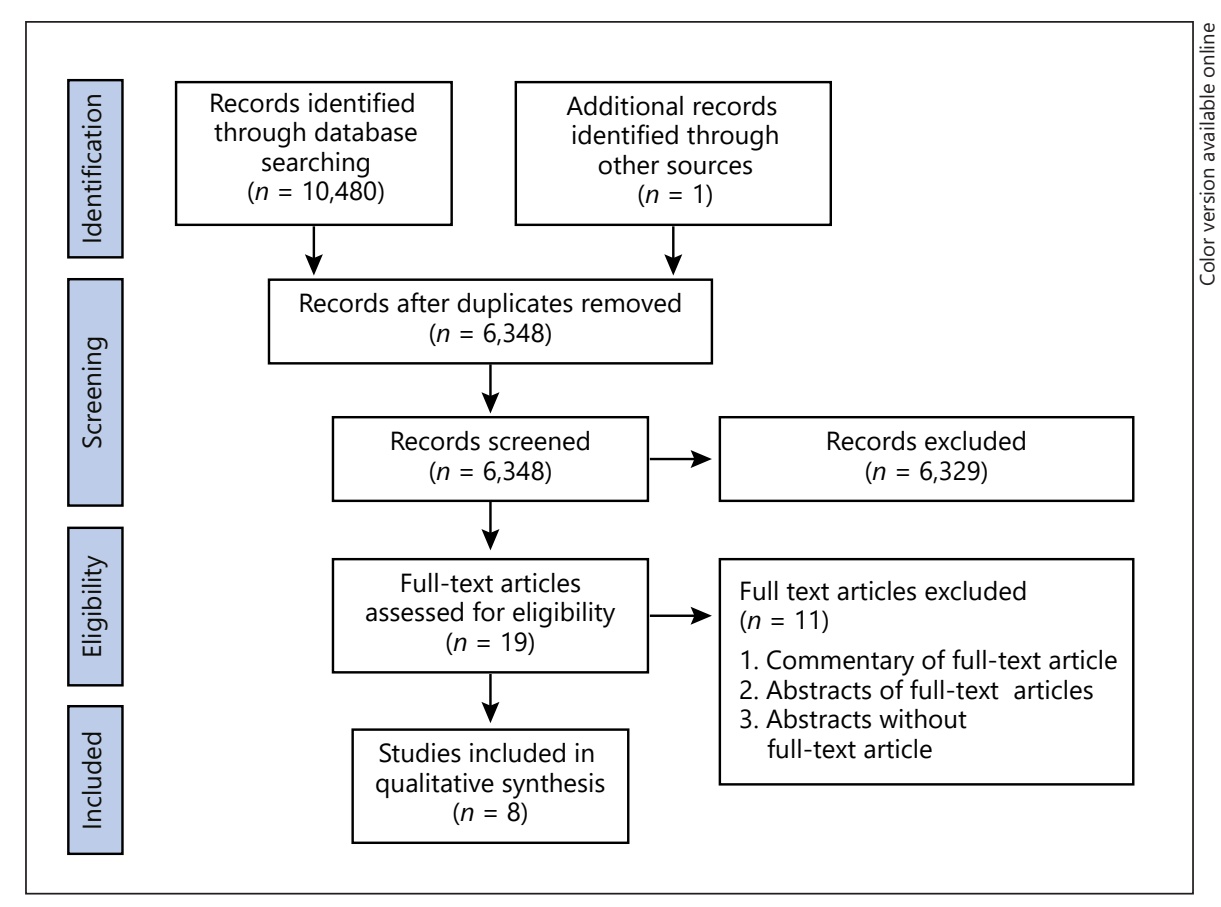

be reached, reports were referred to a third author for arbitration. Outcomes were growth (weight, height, and occipital-frontal head circumference), metabolic health (body mass index, adiposity, glucose metabolism, diabetes, hypertension, and fatty liver), and neurodevelopment (intelligence, processing speed, learning, attention, executive function, behavior, cerebral palsy, vision deficits, and hearing deficits), following discharge from the NICU. Subgroup analyses were completed for varying definitions of hyperglycemia, duration of hyperglycemia, and insulin treatment.

\section{Data Extraction and Analysis}

Data were extracted independently by 2 authors using a customized form (online suppl. 3) including study location, design, population, hyperglycemia definition, hyperglycemia assessment, hyperglycemia incidence, insulin exposure, episodes of insulinrelated hypoglycemia, comparator group, sample size, growth outcomes, metabolic outcomes, neurodevelopmental outcomes, sexspecific outcomes, longitudinal follow-up rates, and study confounders. Principal summary measures were differences in means and correlation coefficients. Quality assessment was independently assessed by 2 authors using the GRADE system [22]. Conflicts were resolved by consensus of all authors.

\section{Results}

\section{Description of Selected Studies}

Of the 6,348 records identified, 6,329 were excluded following title and abstract screening, and a further 11 were excluded following full-text review (Fig. 1). A total of 8 studies involving 987 VLBW infants were included in the analysis $[6,7,23-28]$. The majority of studies focused on the long-term metabolic and neurodevelopmental outcomes of early hyperglycemia versus no hyperglycemia [1, 6, 7, 23-25]. Two studies reported long-term metabolic and neurodevelopmental outcomes following early hyperglycemia intervention (tight vs. standard glycemic control [26], insulin treatment [27]). A meta-analysis of outcomes was not performed given the small number of studies and the heterogeneity of long-term outcomes.

\section{Normoglycemia versus Hyperglycemia}

The design and results of these studies are summarized in Table 2. Hyperglycemia was defined differently between studies using threshold values between 150 (8.3 $\mathrm{mmol} / \mathrm{L})$ and $182 \mathrm{mg} / \mathrm{dL}(10.1 \mathrm{mmol} / \mathrm{L})$ [7, 23-25, 27, 28]. Zamir et al. [28] reported 3 tiers of BG thresholds as follows: >180 (10.0 mmol/L), >216 (12.0 mmol/L), and $>252 \mathrm{mg} / \mathrm{dL}$ (14.0 mmol/L). Three studies reported outcomes following varying duration (number of days) of hyperglycemia $[23,25,28]$. The incidence of hyperglycemia was $45-77 \%$ in studies using a BG threshold of 150 $\mathrm{mg} / \mathrm{dL}(8.3 \mathrm{mmol} / \mathrm{L})$ [23-25] and $8-17.5 \%$ in studies using a BG threshold of $180 \mathrm{mg} / \mathrm{dL}(10.0 \mathrm{mmol} / \mathrm{L})[7,27$, 28].

Six studies compared long-term outcomes following early hyperglycemia. Growth was reported by weight, height, occipital-frontal head circumference percentiles, 


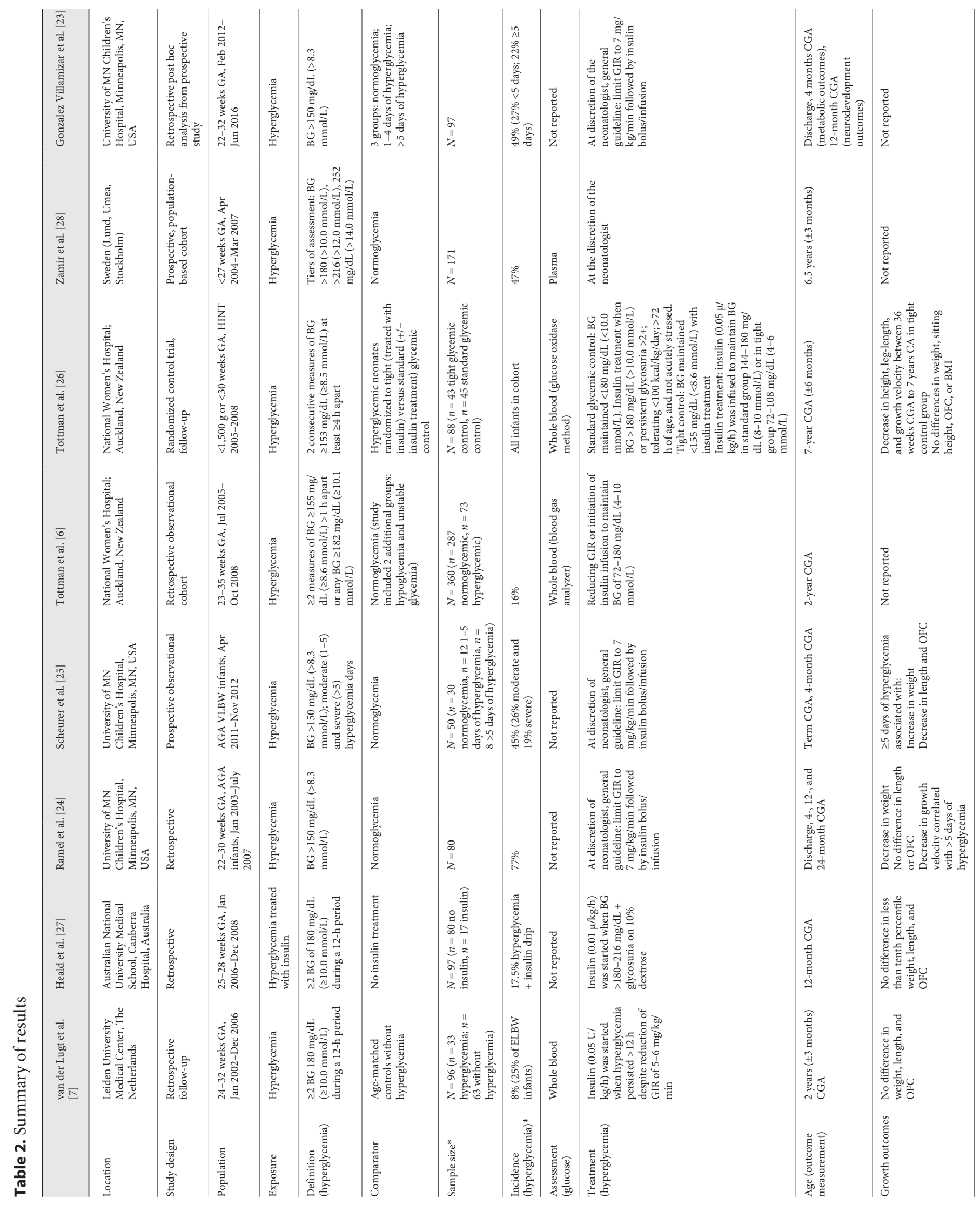




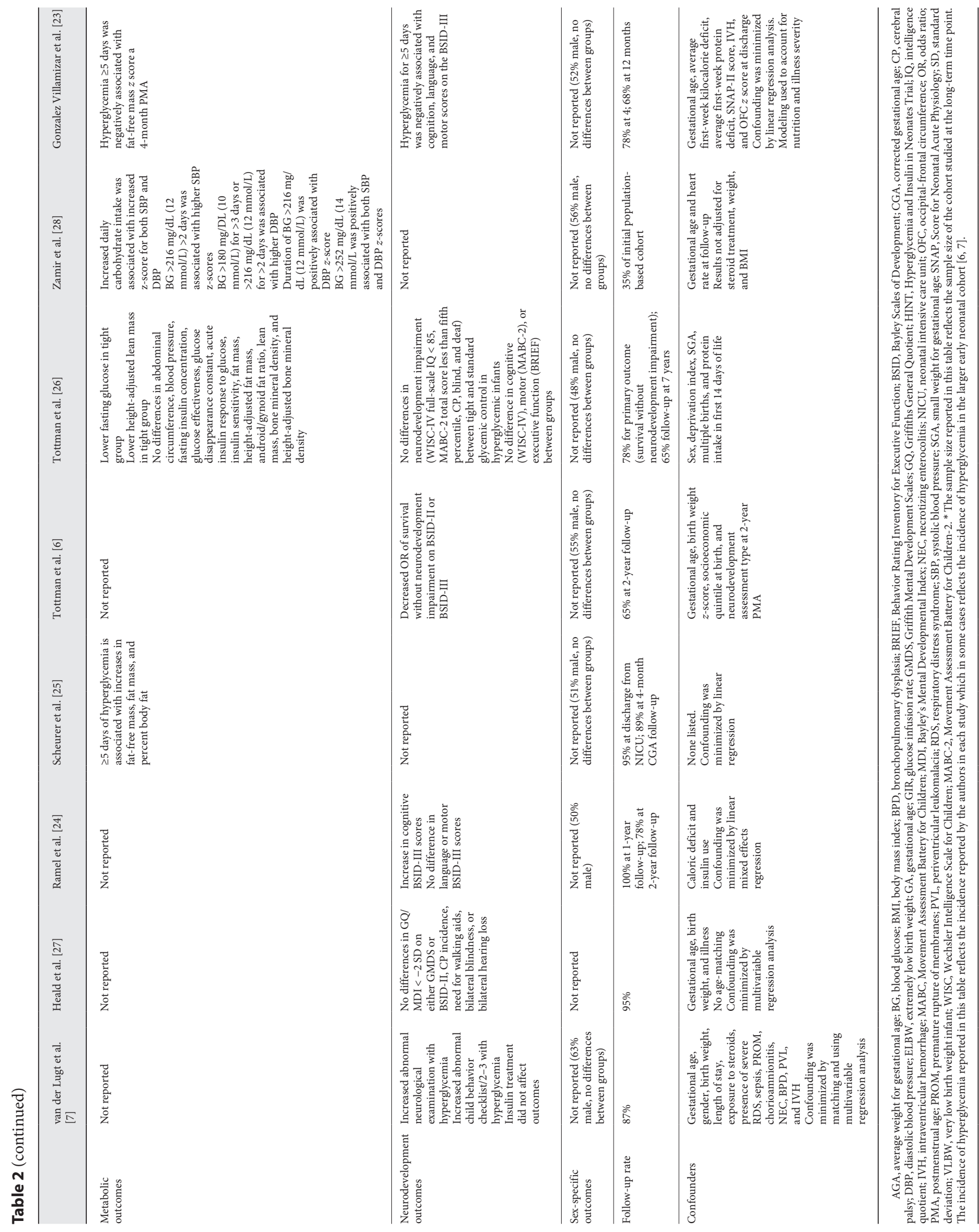


z-scores, or velocity. Three studies reported associations between hyperglycemia in VLBW infants and long-term growth $[7,24,25]$. Metabolic outcomes studied were defined as body composition or blood pressure. Two studies evaluated body composition in VLBW infants with hyperglycemia $[23,25]$. One study evaluated blood pressure in VLBW infants with hyperglycemia at 6.5 years [28]. Collectively, these outcomes were studied between 4-month corrected GA (CGA) and 6.5 years [7, 24, 25]. Four studies evaluated neurodevelopment between 4-months CGA and 2 years of age [7,23-25]. The location of the study, population, study design, comparator group, age the outcome measurement was assessed, and method of assessment of long-term outcome differed between studies.

Treatment of Hyperglycemia: Tight Glycemic Control

Alsweiler et al. [29] performed a randomized controlled trial randomizing preterm infants to tight glycemic control with insulin (target BG 72-108 mg/dL, 4-6 $\mathrm{mmol} / \mathrm{L}$ ) or standard practice (restrictive insulin use, target BG 144-180 mg/dL, 8-10 mmol/L) for neonatal hyperglycemia. Outcomes from this study showed that preterm infants treated with tight glycemic control (BG 72-108 $\mathrm{mg} / \mathrm{dL}, 4-6 \mathrm{mmol} / \mathrm{L}$ ) were exposed to more insulin, had similar carbohydrate intake, had improved weight gain without change in linear growth at 36 weeks postmenstrual age (PMA), and a 3-fold increase in the incidence of hypoglycemia, compared with the standard practice group [29]. Tottman et al. [26] studied growth, metabolic outcomes, and neurobehavior of this cohort at 7 years of age. These results are summarized in Table 2 and suggest differences in metabolic growth.

\section{Treatment of Hyperglycemia: Insulin Treatment}

Insulin treatment was discussed in 7 of the 8 studies [6, 7, 23-27]. In 5 studies, use of insulin was at the discretion of the neonatologist $[6,7,23-27]$. Four studies, 3 of which were from the same center, provided general guidelines that the glucose infusion rate (GIR) was initially limited followed by insulin treatment if hyperglycemia persisted $[6,23-25]$. Five studies provided descriptive information and addressed complications related to or outcomes associated with insulin treatment $[7,24-27]$.

The remaining 4 studies described insulin use in the hyperglycemia group [7,24-26]. No study found associations between insulin use and growth, metabolic, or neurodevelopment outcomes [7, 24-26]. Van der Lugt et al. [7] reported the mean duration of insulin infusion as 129 $\mathrm{h}$ with a mean infusion rate of $0.06 \mathrm{U} / \mathrm{kg} / \mathrm{h}$. Scheurer et al. [25] reported increased use of insulin with longer duration of hyperglycemia. VLBW infants with 1-5 days of hyperglycemia had a mean of 2.6 days of insulin treatment compared with infants with 5 or more days of hyperglycemia who had a mean of 15.7 days of insulin treatment [25]. Ramel et al. [24] reported both duration of insulin treatment and its association with outcome measurements. The authors described a mean of 2.14 insulin days for treatment of hyperglycemia and reported no association between insulin treatment and growth or neurodevelopment outcomes [24]. Two of these studies reported 0.3 and 0.65 mean number of episodes of hypoglycemia but did not report associations between hypoglycemia and outcomes [7, 24].

Heald et al. [27] aimed to study the effects of insulin infusion for the treatment of early hyperglycemia in preterm infants on long-term neurodevelopmental outcome. Infants with hyperglycemia (BG $>180 \mathrm{mg} / \mathrm{dL}, 10 \mathrm{mmol} / \mathrm{L}$ ) met criteria for insulin treatment when treatment for hyperglycemia limited nutrition to $10 \%$ dextrose infusion, and the infant had persistent glucosuria [27]. Of the infants who received insulin, the median $B G$ prior to insulin infusion was $223 \mathrm{mg} / \mathrm{dL}$ (12.4 mmol/L) [27]. The median length of insulin treatment was $41 \mathrm{~h}$. The authors reported $1.3 \%$ episodes of hypoglycemia with insulin treatment which occurred in 2 of 17 infants in the insulin-treated group [27]. These researchers found no significant differences in infant growth failure, Bayley Scales of Infant Development (BSID-II) motor scores, incidence of cerebral palsy, need for walking aids, blindness, or hearing loss in VLBW infants with hyperglycemia with or without insulin treatment at 12-month CGA [27].

\section{Quality of Evidence}

The overall quality of evidence, using established GRADE criteria, is summarized in Table 3 [22]. Overall, the quality of evidence to characterize associations between hyperglycemia and long-term outcomes was low. The quality of evidence demonstrating an association between duration of early hyperglycemia and long-term outcomes was moderate. The quality of evidence that a BG threshold of $150 \mathrm{mg} / \mathrm{dL}(8.3 \mathrm{mmol} / \mathrm{L})$, a surrogate definition for severity of hyperglycemia, in VLBW infants is associated with worse long-term outcomes was moderate. Last, the quality of evidence reporting both complications and effects of insulin treatment for early hyperglycemia on long-term outcomes of VLBW infants was low.

Evidence reported as very low- or low-grade quality were limited by the number of studies reporting an out- 


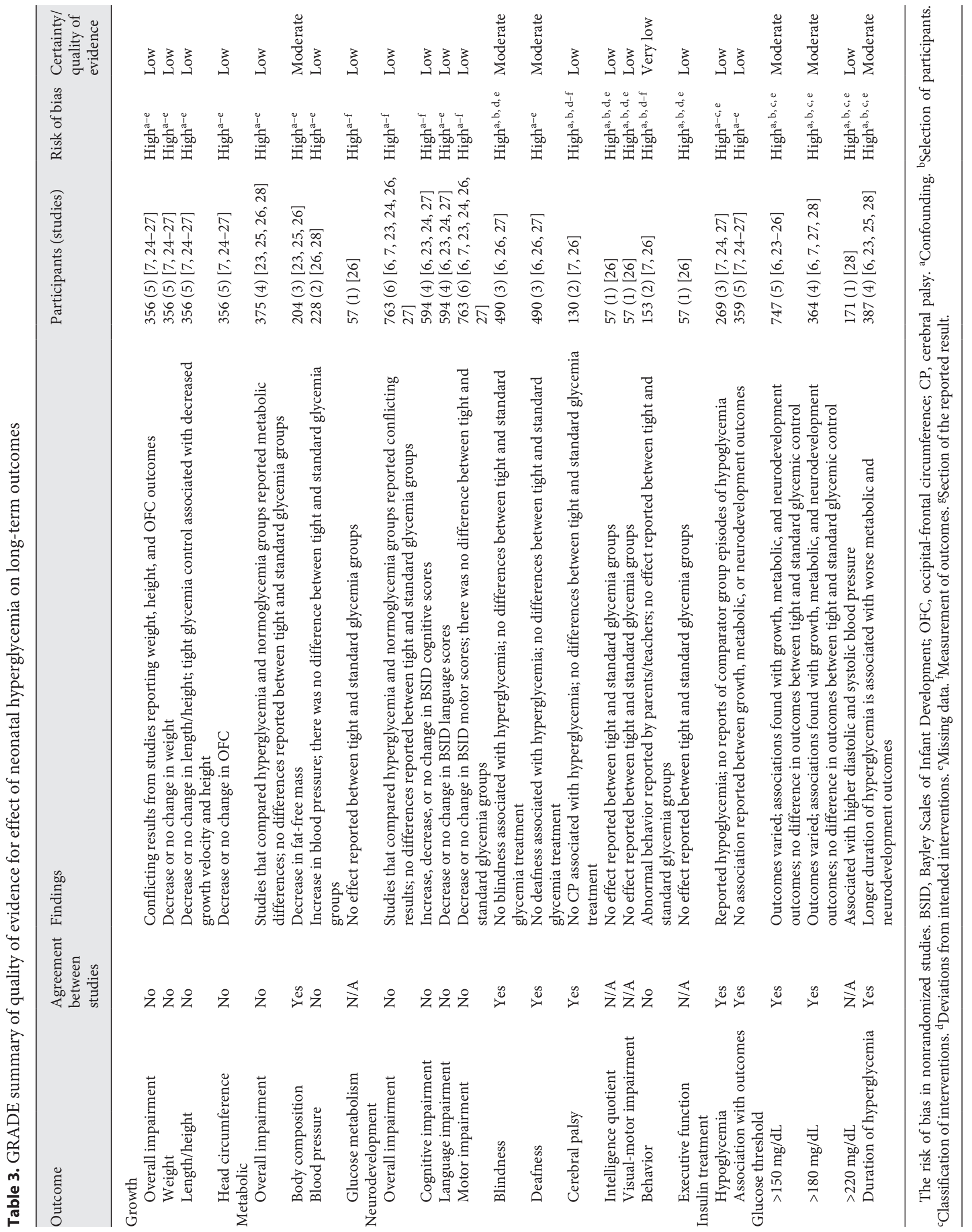


come or reported conflicting results. Collectively, studies were underpowered across growth, metabolic, and neurodevelopment outcomes to interpret findings confidently. All studies showed a risk of bias limiting the quality of evidence. Some studies did not adjust for confounders. All studies adjusting for confounders did not assess the same confounders. Additionally, there was variation in BG threshold to define hyperglycemia among the studies as well as variation in the treatment of hyperglycemia both within and between studies. There was an overlap between studies from the same centers, potentially limiting generalizability of the results. Last, studies did not account for sex-specific outcomes. There was one follow-up study from a randomized control trial that initially was considered high-quality due to its randomized study design; however, this study received a lower quality grade given its small sample size and lack of a normoglycemia comparator group [26].

\section{Discussion}

Best management practices for hyperglycemia in the VLBW preterm infant remain unknown despite its common occurrence in this population. Our findings report the results of a systematic search for all relevant studies on long-term growth, metabolic health, and neurodevelopmental outcomes after early neonatal hyperglycemia in the VLBW preterm infant. The primary objective of this study was to determine if there was enough evidence to guide clinical management of early hyperglycemia in preterm infants. A meta-analysis, which would have guided interpretation of the existing findings, was not conducted because of the clinical and methodologic heterogeneity in a small number of published studies. Additionally, the quality of evidence presented in the collective studies is low. Therefore, currently, there is not enough high-quality evidence of long-term outcomes to guide clinical management of early hyperglycemia in the preterm infant.

We critically evaluated the quality of evidence to determine if (1) hyperglycemia affects growth, metabolic health, and neurodevelopmental outcomes following NICU discharge of VLBW preterm infants; (2) the severity and duration of hyperglycemia affects long-term outcomes in VLBW preterm infants after hyperglycemia; and (3) treatment of hyperglycemia attenuated adverse long-term outcomes. The results are conflicting. Some studies found differences in long-term outcomes between VLBW preterm infants with hyperglycemia and without hyperglycemia, while other studies found no differences between groups.

Studies were heterogeneous and underpowered to adequately determine differences between groups. There appears to be a trend among the studies that the severity and duration of hyperglycemia are associated with worse metabolic or neurodevelopmental outcomes. However, without adequately powered studies aimed at investigating this trend, clinicians are left with a hypothesis rather than relevant conclusions to inform clinical decisionmaking. Treatment of hyperglycemia with insulin was not associated with a change in long-term outcomes. The threshold for treatment, the infant's nutritional status, and protocols for insulin use varied among the studies or were not reported, thus confounding interpretation to guide evidence-based practices. In addition to standardized experimental designs with consistent variables, we recommend the following considerations in hyperglycemia assessment, outcome analysis, and treatment in future research.

\section{Hyperglycemia Assessment}

The assessment of hyperglycemia was not standardized within or between institutions. In future study development, we recommend standardized hyperglycemia screening, evaluation, and definition. First, infants who are sicker or diagnosed to have glucose disturbances generally have more frequent glucose measurements than clinically stable infants with euglycemia. The differences in frequency of glucose evaluation may confound longterm outcomes; therefore, frequency should be determined a priori. Second, details of how BG concentration is measured should be reported in each study. BG values can vary as much as $20 \%$ depending on the specimen (i.e., whole blood vs. plasma) and assessment method, thereby confounding potential conclusions regarding early hyperglycemia and long-term outcome associations [30]. Third, we recommend a unified definition of hyperglycemia. There was moderate quality of evidence to suggest that a $\mathrm{BG}$ of $>150 \mathrm{mg} / \mathrm{dL}(>8.3 \mathrm{mmol} / \mathrm{L})$ was associated with long-term adverse metabolic and neurodevelopmental outcomes, and there was no difference in outcomes whether hyperglycemia was defined as 150 (8.3 $\mathrm{mmol} / \mathrm{L})$ or $180 \mathrm{mg} / \mathrm{dL}(10.0 \mathrm{mmol} / \mathrm{L})$. Therefore, our recommendation is to use a $B G$ threshold of $150 \mathrm{mg} / \mathrm{dL}$ $(8.3 \mathrm{mmol} / \mathrm{L})$.

\section{Outcome Analysis}

The impact of preterm birth, postnatal growth velocity, exposure to over/undernutrition, insulin treatment, 
and sex are potential drivers of developmental programming of metabolic disease and neurodevelopmental impairment in adulthood. For example, male preterm infants are at a higher risk for mortality and morbidity compared with female infants [31]. Increased attention to metabolic and neurodevelopment outcomes, both of which are especially susceptible to hyperglycemia-mediated programming in adults born preterm and sex differences within outcomes will inform current treatment practices.

The most common metabolic outcome in individuals born preterm is higher blood pressure [32]. Prematurity also increases the risk of short stature [33], insulin resistance and diabetes $[34,35]$, increased visceral fat mass [36], and cardiac hypertrophy [37]. Adult dyslipidemia, coronary artery, and cerebrovascular diseases in infants born preterm have shown mixed findings across studies [32]. Preclinical studies evaluating hyperglycemia-induced programming of adult metabolic disease provide support that early metabolic stress permanently alters tissue and organ function and predisposes to metabolic disease $[38,39]$. Important metabolic outcomes should include linear growth/height, body composition (\% fat mass, fat-free mass), fasting glucose, and blood pressure.

Preterm birth increases the risk for psychiatric disorders, social-emotional challenges, and cognitive impairments [32]. Research reveals a multifactorial psychopathology for these associations [32]. Impaired postnatal growth is associated with delayed brain development and diminished cortical connections with persistent structural and functional changes on neuroimaging in adulthood [40,41]. Additionally, both clinical and preclinical studies have shown that hyperglycemia alters brain structure and is associated with worse neurodevelopmental outcomes $[42,43]$. Key neurodevelopmental outcomes should focus on determinants of disability, including motor, vision, cognitive, and social-emotional assessments.

None of the studies in this analysis reported sex-specific outcomes. All but 1 study reported no significant differences in males in the control and hyperglycemia groups $[6,7,23-26,28]$. The Fenton growth chart accounts for sex differences in growth of preterm infants [44]. Variation in growth rates of males and females may suggest that nutritional requirements, and therefore, the incidence of hyperglycemia may be sex-specific. Research on sex-specific nutritional guidelines is sparse in preterm infants [45]. Studies have shown that early nutritional interventions have a pronounced neurodevelopmental benefit in preterm males compared to female infants [46,

Long-Term Outcomes in VLBW Infant

Hyperglycemia
47]. Additionally, 1 study has shown that female preterm infants have higher insulin secretion at similar BG concentrations than that of male preterm infants [48]. Collectively, these studies suggest that incidence of early hyperglycemia and the effect of its treatment on long-term outcomes differ between male and female infants. Such sex-specific results should be reported in future studies.

\section{Treatment of Hyperglycemia}

There are variations in management practices of hyperglycemia. The most widely practiced treatment strategy is a reduction in GIR. Although this practice is effective in achieving euglycemia [49], it can lead to lower energy intake early in the NICU course, which is associated with worse metabolic and neurodevelopmental outcomes $[6,17,24,26,50,51]$. Insulin administration is also frequently used for treating hyperglycemia and is effective in lowering BG. Insulin treatment, in contrast to GIR reduction, also promotes weight gain in VLBW infants [49]. The use of insulin in the treatment of hyperglycemia in VLBW infants is controversial because of its potential to cause hypoglycemia and impact on long-term metabolic outcomes. To date, there have been no randomized controlled trials that have investigated long-term growth, metabolic, or neurodevelopment outcomes in preterm infants with early hyperglycemia treated with or without insulin.

There were only 2 studies aimed at investigating the effect of intervention for early hyperglycemia (tight glycemic control and insulin treatment) on long-term metabolic and neurodevelopmental outcomes in preterm infants [26, 27]. Tottman et al. [26] longitudinally followed a cohort of previously randomized preterm infants to tight versus standard glycemic control. The study included all surviving infants from the Hyperglycemia in Neonates Trial which was powered to detect difference in outcome (linear growth rate) between infants randomized to standard or tight glycemic control at 36 weeks PMA rather than 7-year outcomes [26, 29]. We estimate a sample size of 775 , rather than the reported sample size of 88 , is necessary to detect a difference of $10 \%$ effect size between groups in neurodevelopmental impairment at $80 \%$ power and an alpha level of 0.05 . An example of this potential limitation is that the adjusted odds ratio for mean fullscale intelligence quotient $<85$ was $0.75(0.25-2.2195 \%$ $\mathrm{CI})$ and for motor impairment $0.74(0.25-2.2095 \% \mathrm{CI})$ if treated with tight glycemic control [26]. These findings were not statistically significant but may be clinically significant if adequately powered to detect differences between groups. 
Table 4. Proposed study design: long-term metabolic and neurodevelopmental outcomes following early hyperglycemia treatment in preterm infants receiving standardized nutrition

\begin{tabular}{|c|c|}
\hline Study population & VLBW infants $(<32$ weeks or $<1,500 \mathrm{~g})$ \\
\hline Study design & Multicenter prospective international cohort and randomized control trial \\
\hline Treatment randomization & $\begin{array}{l}\text { Reduction of GIR to basal metabolic caloric needs followed by insulin versus no nutritional modification } \\
\text { with insulin }\end{array}$ \\
\hline Treatment threshold & BG over $180 \mathrm{mg} / \mathrm{dL}(>10 \mathrm{mmol} / \mathrm{L}) \geq 4 \mathrm{~h}$ during the first 2 weeks of life \\
\hline Assessment (glucose) & Continuous glucose monitoring \\
\hline Nutrition & $\begin{array}{l}\text { Standardized parenteral and enteral nutrition and vitamin supplementation during the first } 2 \text { weeks of } \\
\text { life }\end{array}$ \\
\hline Primary outcome & Survival without significant metabolic or neurodevelopmental impairment at 5 years of age* \\
\hline Secondary outcomes & $\begin{array}{l}\text { Individual components of primary outcome, anthropometrics, BSID-III, need for glasses, body } \\
\text { composition, glucose metabolism, and quality of life measurements }\end{array}$ \\
\hline Modifying variables & Insulin treatment, sex, and duration/severity of hyperglycemia \\
\hline Confounding variables & $\begin{array}{l}\text { Maternal characteristics, demographic characteristics, infant characteristics, morbidities associated with } \\
\text { preterm birth, food consumption, social determinants of health, and hypoglycemia }\end{array}$ \\
\hline
\end{tabular}

VLBW, very low birth weight; BSID, Bayley Scales of Infant Development; BG, blood glucose; GIR, glucose infusion rate; PMA, postmenstrual age. * Any of the following: $\mathrm{BMI} \geq 85$ percentile, fasting glucose $\geq 100 \mathrm{mg} / \mathrm{dL}, \mathrm{HbAlc} \geq 5.7 \%$, sBP or $\mathrm{dBP}>95$ percentile, cerebral palsy, Movement Assessment Battery for Children-2 total score less than fifth percentile, hearing impairment requiring hearing aids, visual acuity of 6/60, full-scale IQ, standard score $>1$ standard deviation below the mean, visual acuity of 6/60 or worse in best eye, $\mathrm{ADHD} / \mathrm{Mental}$ health diagnosis requiring behavior/psychological therapy, early intervention, or medication.

Heald et al. [27] retrospectively studied treatment-associated hypoglycemia and neurodevelopmental outcomes at 12-month PMA in preterm infants with hyperglycemia treated with insulin. This study has several factors limiting its generalizability. First, the study is retrospective. Second, the study is limited by unequal and small sample sizes ( $n=64$ no treatment, $n=9$ insulin treatment) and not powered to detect differences in outcomes between the groups. For example, there was $>10 \%$ difference in the number of infants with weight and height less than tenth percentile in no treatment and insulin treatment groups. These differences were not significant potentially due to the small sample size. Third, infants who received insulin were of lower birth weight and GA.
All studies reporting insulin use in the treatment of hyperglycemia did not show associations between insulin treatment and long-term growth, metabolic, or neurodevelopmental outcomes $[6,7,23,24,27]$. Given that less mature and more critically ill infants are more likely to have hyperglycemia, and thus more likely to be treated with insulin, the collective finding showing no difference in long-term outcomes between infants treated or not treated with insulin may suggest a protective effect of insulin treatment on long-term metabolic and neurodevelopmental outcomes. None of these studies, however, were adequately powered to provide clinical direction regarding insulin treatment in early hyperglycemia for preterm infants.

An established concern of insulin treatment is its association with hypoglycemia in the VLBW population, 
which has previously been reported to occur $>25 \%$ of the time [49]. The results from the studies in this analysis however would suggest this is an overestimation of hypoglycemia episodes associated with insulin treatment [7, 24,27 . None of the studies in this analysis included insulin-associated hypoglycemia as a mediator of longterm outcomes. Improved reporting of insulin-associated hypoglycemia, as well as including hypoglycemia episodes as a confounder in analyses, are necessary, given the evidence that persistent hypoglycemia is associated with adverse neurodevelopmental outcomes [52].

Caloric deficit, a potential result of decreased GIR as treatment of hyperglycemia, was associated with longterm growth, metabolic, and neurodevelopmental compromise [1, 23-25]. Most recently, Gonzalez Villamizar et al. [23] assessed the potential influence of limiting early nutrition as a treatment of hyperglycemia in VLBW infants. This group reported lower BSID-III cognition, language, and motor scores in VLBW infants exposed to hyperglycemia ( $B G>150 \mathrm{mg} / \mathrm{dL}, 8.3 \mathrm{mmol} / \mathrm{L}$ ). However, when the analysis model was adjusted for average firstweek kilocalorie and protein deficits, the association between hyperglycemia and BSID-III language and cognitive scores resolved [23]. This study emphasizes the importance of providing optimal early nutrition even in the setting of hyperglycemia. Future studies that are powered to evaluate different treatment strategies (i.e., reduction in GIR and/or insulin treatment) are needed to inform best practice in the treatment of hyperglycemia.

\section{Conclusions}

Current evidence of long-term health outcomes following early hyperglycemia in VLBW infants does not provide high-quality recommendations to guide assessment and treatment of early hyperglycemia. There is a critical need, as well as clinical equipoise, for well-designed, large randomized controlled trials and prospective studies that address the following questions: (1) who is at risk for long-term metabolic and neurodevelopmental compromise after hyperglycemia? (2) what BG threshold and duration predisposes VLBW infants to worse metabolic and neurodevelopmental outcomes? and (3) which interventions decrease the risk of long-term metabolic and neurodevelopmental compromise after hyperglycemia in VLBW infants? Prospective studies using standardized approaches to early nutrition, evaluation and treatment of hyperglycemia, and outcome assessment will improve the quality of evidence to inform clin- ical practice for management of hyperglycemia. We propose, and have included, an example of such a study (Table 4). We invite content experts and clinicians worldwide to collaborate on such studies and a prospective metaanalysis.

\section{Statement of Ethics}

An ethics statement is not applicable because this study is based exclusively on published literature.

\section{Conflict of Interest Statement}

The authors have no conflicts of interest to declare.

\section{Funding Sources}

Dr. Paulsen was supported by the National Institute of Health Building Interdisciplinary Research Careers in Women's Health grant HD055887. The NIH had no role in the design and conduct of the study.

\section{Author Contributions}

M.E.P., S.J.B., and R.B.R. conceptualized the study. M.E.P., S.J.B., J.M.S., S.E.R., and R.B.R. designed the study. S.J.B. developed the search strategy and co-ordinated and supervised data collection. M.E.P., S.E.R., and R.B.R. independently reviewed abstracts to determine their eligibility for inclusion. M.E.P. and K.M.S. analyzed and determined the quality of studies. M.E.P. and S.J.B. drafted the initial manuscript. M.E.P. revised the manuscript. K.M.S., J.M.S., S.E.R., and R.B.R. critically reviewed and revised the manuscript. All the authors approved the final manuscript as submitted and agree to be accountable for all aspects of the work.

$\begin{array}{ll}1 \text { Zeferences } & \text { Zamir I, Tornevi A, Abrahamsson T, Ahlsson } \\ & \text { F, Engström E, Hallberg B, et al. Hyperglyce- } \\ & \text { mia in extremely preterm infants-insulin } \\ & \text { treatment, mortality and nutrient intakes. J } \\ & \text { Pediatr. 2018;200:104-10.e1. } \\ 2 & \text { Beardsall K, Vanhaesebrouck S, Ogilvy-Stuart } \\ & \text { AL, Vanhole C, Palmer CR, Ong K, et al. Prev- } \\ & \text { alence and determinants of hyperglycemia in } \\ & \text { very low birth weight infants: cohort analyses } \\ & \text { of the NIRTURE study. J Pediatr. 2010; } \\ & 157(5): 715-9 . e 1-3 . \\ 3 & \text { Beardsall K, Vanhaesebrouck S, Ogilvy-Stuart } \\ & \text { AL, Vanhole C, Palmer CR, van Weissen- } \\ & \text { bruch M, et al. Early insulin therapy in very- } \\ & \text { low-birth-weight infants. N Engl J Med. 2008; } \\ & 359(18): 1873-84 .\end{array}$


4 Blanco CL, Baillargeon JG, Morrison RL, Gong AK. Hyperglycemia in extremely low birth weight infants in a predominantly Hispanic population and related morbidities. J Perinatol. 2006;26(12):737-41.

5 Yoon JY, Chung HR, Choi CW, Yang SW, Kim BI, Shin CH. Blood glucose levels within 7 days after birth in preterm infants according to gestational age. Ann Pediatr Endocrinol Metab. 2015;20(4):213-9.

6 Tottman AC, Alsweiler JM, Bloomfield FH, Pan M, Harding JE. Relationship between measures of neonatal glycemia, neonatal illness, and 2-year outcomes in very preterm infants. J Pediatr. 2017;188:115-21.

7 van der Lugt NM, Smits-Wintjens VE, van Zwieten $\mathrm{PH}$, Walther FJ. Short and long term outcome of neonatal hyperglycemia in very preterm infants: a retrospective follow-up study. BMC Pediatr. 2010;10:52.

8 Szymonska I, Jagla M, Starzec K, Hrnciar K, Kwinta P. The incidence of hyperglycaemia in very low birth weight preterm newborns. Results of a continuous glucose monitoring study: preliminary report. Dev Period Med. 2015;19(3 Pt 1):305-12.

9 Alexandrou G, Skiöld B, Karlén J, Tessma MK, Norman M, Adén U, et al. Early hyperglycemia is a risk factor for death and white matter reduction in preterm infants. Pediatrics. 2010;125(3):e584-91.

10 Hays SP, Smith EO, Sunehag AL. Hyperglycemia is a risk factor for early death and morbidity in extremely low birth-weight infants. Pediatrics. 2006;118(5):1811-8.

11 Kao LS, Morris BH, Lally KP, Stewart CD, Huseby V, Kennedy KA. Hyperglycemia and morbidity and mortality in extremely low birth weight infants. J Perinatol. 2006;26(12): 730-6.

12 Georgieff MK. The effect of maternal diabetes during pregnancy on the neurodevelopment of offspring. Minn Med. 2006;89(3):44-7.

13 Adane AA, Mishra GD, Tooth LR. Diabetes in pregnancy and childhood cognitive development: a systematic review. Pediatrics. 2016; 137(5):e20154234.

14 Landi SN, Radke S, Engel SM, Boggess K, Stürmer T, Howe AS, et al. Association of long-term child growth and developmental outcomes with metformin vs insulin treatment for gestational diabetes. JAMA Pediatr. 2019;173(2):160-8.

15 Logan KM, Gale C, Hyde MJ, Santhakumaran S, Modi N. Diabetes in pregnancy and infant adiposity: systematic review and meta-analysis. Arch Dis Child Fetal Neonatal Ed. 2017; 102(1):F65-72.

16 Hay WW Jr. Nutritional support strategies for the preterm infant in the neonatal intensive care unit. Pediatr Gastroenterol Hepatol Nutr. 2018;21(4):234-47.

17 Hay WW Jr. Aggressive Nutrition of the Preterm Infant. Curr Pediatr Rep. 2013;1(4).

18 Ramel S, Rao R. Hyperglycemia in extremely preterm infants. Neoreviews. 2020;21(2):e8997.
19 Moher D, Liberati A, Tetzlaff J, Altman DG, Group P. Preferred reporting items for systematic reviews and meta-analyses: the PRISMA statement. J Clin Epidemiol. 2009;62(10): 1006-12.

20 Richardson WS, Wilson MC, Nishikawa J, Hayward RS. The well-built clinical question: a key to evidence-based decisions. ACP J Club. 1995 Nov-Dec;123(3):A12-3.

21 Ouzzani M, Hammady H, Fedorowicz Z, Elmagarmid A. Rayyan-a web and mobile app for systematic reviews. Syst Rev. 2016;5(1): 210.

22 Balshem H, Helfand M, Schünemann HJ, Oxman AD, Kunz R, Brozek J, et al. GRADE guidelines: 3 . Rating the quality of evidence. J Clin Epidemiol. 2011;64(4):401-6.

23 Gonzalez Villamizar JD, Haapala JL, Scheurer JM, Rao R, Ramel SE. Relationships between early nutrition, illness, and later outcomes among infants born preterm with hyperglycemia. J Pediatr. 2020:223;29-33.e2.

24 Ramel SE, Long JD, Gray H, Durrwachter-Erno K, Demerath EW, Rao R. Neonatal hyperglycemia and diminished long-term growth in very low birth weight preterm infants. J Perinatol. 2013;33(11):882-6.

25 Scheurer JM, Gray HL, Demerath EW, Rao R, Ramel SE. Diminished growth and lower adiposity in hyperglycemic very low birth weight neonates at 4 months corrected age. J Perinatol. 2016;36(2):145-50.

26 Tottman AC, Alsweiler JM, Bloomfield FH, Gamble G, Jiang Y, Leung M, et al. Long-term outcomes of hyperglycemic preterm infants randomized to tight glycemic control. J Pediatr. 2018;193:68-75.e1.

27 Heald A, Abdel-Latif ME, Kent AL. Insulin infusion for hyperglycaemia in very preterm infants appears safe with no effect on morbidity, mortality and long-term neurodevelopmental outcome. J Matern Fetal Neonatal Med. 2012;25(11):2415-8.

28 Zamir I, Stoltz Sjöström E, Edstedt Bonamy AK, Mohlkert LA, Norman M, Domellöf M. Postnatal nutritional intakes and hyperglycemia as determinants of blood pressure at 6.5 years of age in children born extremely preterm. Pediatr Res. 2019;86(1):115-21.

29 Alsweiler JM, Harding JE, Bloomfield FH. Tight glycemic control with insulin in hyperglycemic preterm babies: a randomized controlled trial. Pediatrics. 2012;129(4):639-47.

30 Kim HS. Blood glucose measurement: is serum equal to plasma? Diabetes Metab J. 2016; 40(5):365-6.

31 Boghossian NS, Geraci M, Edwards EM, Horbar JD. Sex differences in mortality and morbidity of infants born at less than 30 weeks' gestation. Pediatrics. 2018;142(6):e20182352.

32 Raju TNK, Pemberton VL, Saigal S, Blaisdell CJ, Moxey-Mims M, Buist S, et al. Long-term healthcare outcomes of preterm birth: an executive summary of a conference sponsored by the National Institutes of Health. J Pediatr. 2017;181:309-18.e1.
33 Saigal S, Stoskopf B, Streiner D, Paneth N, Pinelli J, Boyle M. Growth trajectories of extremely low birth weight infants from birth to young adulthood: a longitudinal, population-based study. Pediatr Res. 2006;60(6): 751-8.

34 Kajantie E, Strang-Karlsson S, Hovi P, Wehkalampi K, Lahti J, Kaseva N, et al. Insulin sensitivity and secretory response in adults born preterm: the helsinki study of very low birth weight adults. J Clin Endocrinol Metab. 2015;100(1):244-50.

35 Hofman PL, Regan F, Jackson WE, Jefferies C, Knight DB, Robinson EM, et al. Premature birth and later insulin resistance. $\mathrm{N}$ Engl J Med. 2004;351(21):2179-86.

36 Thomas EL, Parkinson JR, Hyde MJ, Yap IK, Holmes E, Doré CJ, et al. Aberrant adiposity and ectopic lipid deposition characterize the adult phenotype of the preterm infant. Pediatr Res. 2011;70(5):507-12.

37 Lewandowski AJ, Augustine D, Lamata P, Davis EF, Lazdam M, Francis J, et al. Preterm heart in adult life: cardiovascular magnetic resonance reveals distinct differences in left ventricular mass, geometry, and function. Circulation. 2013;127(2):197-206.

38 Paulsen ME, Rosario FJ, Wesolowski SR, Powell TL, Jansson T. Normalizing adiponectin levels in obese pregnant mice prevents adverse metabolic outcomes in offspring. FASEB J. 2019;33(2):2899-909.

39 Vaughan OR, Rosario FJ, Powell TL, Jansson T. Normalisation of circulating adiponectin levels in obese pregnant mice prevents cardiac dysfunction in adult offspring. Int J Obes. 2020;44(2):488-99.

40 Bauml JG, Daamen M, Meng C, Neitzel J, Scheef L, Jaekel J, et al. Correspondence between aberrant intrinsic network connectivity and gray-matter volume in the ventral brain of preterm born adults. Cereb Cortex. 2015; 25(11):4135-45.

41 Jurcoane A, Daamen M, Scheef L, Bäuml JG, Meng C, Wohlschläger AM, et al. White matter alterations of the corticospinal tract in adults born very preterm and/or with very low birth weight. Hum Brain Mapp. 2016;37(1): 289-99.

42 Ramel SE, Haapala J, Super J, Boys C, Demerath EW. Nutrition, illness and body composition in very low birth weight preterm infants: implications for nutritional management and neurocognitive outcomes. Nutrients. 2020; 12(1):145.

43 Satrom KM, Ennis K, Sweis BM, Matveeva TM, Chen J, Hanson L, et al. Neonatal hyperglycemia induces CXCL10/CXCR3 signaling and microglial activation and impairs longterm synaptogenesis in the hippocampus and alters behavior in rats. J Neuroinflamm. 2018; 15(1):82.

44 Fenton TR, Kim JH. A systematic review and meta-analysis to revise the Fenton growth chart for preterm infants. BMC Pediatr. 2013; 13:59. 
45 Alur P. Sex differences in nutrition, growth, and metabolism in preterm infants. Front Pediatr. 2019;7:22.

46 Isaacs EB, Fischl BR, Quinn BT, Chong WK, Gadian DG, Lucas A. Impact of breast milk on intelligence quotient, brain size, and white matter development. Pediatr Res. 2010;67(4): 357-62.

47 Poindexter BB, Langer JC, Dusick AM, Ehrenkranz RA; National Institute of Child Health, Human Development Neonatal Research Network. Early provision of parenteral amino acids in extremely low birth weight infants: relation to growth and neurodevelop- mental outcome. J Pediatr. 2006;148(3):3005.

48 Dickson JL, Chase JG, Pretty CG, Gunn CA, Alsweiler JM. Hyperglycaemic preterm babies have sex differences in insulin secretion. Neonatology. 2015;108(2):93-8.

49 Bottino M, Cowett RM, Sinclair JC. Interventions for treatment of neonatal hyperglycemia in very low birth weight infants. Cochrane Database Syst Rev. 2011;(10): CD007453.

50 Stephens BE, Walden RV, Gargus RA, Tucker R, McKinley L, Mance M, et al. First-week protein and energy intakes are associated with 18-month developmental outcomes in extremely low birth weight infants. Pediatrics. 2009; 123(5):1337-43.

51 Ehrenkranz RA, Dusick AM, Vohr BR, Wright LL, Wrage LA, Poole WK. Growth in the neonatal intensive care unit influences neurodevelopmental and growth outcomes of extremely low birth weight infants. Pediatrics. 2006;117(4):1253-61.

52 Boluyt N, van Kempen A, Offringa M. Neurodevelopment after neonatal hypoglycemia: a systematic review and design of an optimal future study. Pediatrics. 2006;117(6):223143. 